\title{
INFECÇÃO DE SÍTIO CIRÚRGICO: ESTUDO PROSPECTIVO DE 2.149 PACIENTES OPERADOS
}

\author{
SURGICAL SITE INFECTIONS: PROSPECTIVE STUDY OF 2.149 SURGICAL PATIENTS
}

\author{
Francisco Ney Lemos, TCBC-CE ${ }^{1}$ \\ Adriano Veras Oliveira ${ }^{2}$ \\ Maria Geciliane de Sousa $^{3}$
}

\begin{abstract}
RESUMO: A atual caracterização de infecção do sítio cirúrgico em incisional superficial, incisional profunda e órgão cavidade, em substituição à tradicional definição de "infecção de ferida operatória", associada a estratificação dos pacientes em grupos de risco de infecção cirúrgica de acordo com a metodologia NNISS (National Nosocomial Infection Surveillance System), permitiram a obtenção de taxas de infecção mais fidedignas e estudos comparativos entre instituições diferentes. Baseado nessa metodologia, o presente trabalho analisa prospectivamente 2.149 pacientes operados no Serviço de Cirurgia do Hospital Geral César Cals (HGCC)-CE, estratificados pelo IRIC (Índice de Risco de Infecção Cirúrgica) e comprova diferenças estatisticamente significativas nas taxas de infecção de sítio cirúrgico para os grupos de IRIC 0,1, 2 e 3, respectivamente de $3,2 \%, 7,4 \%, 16,6 \%$ e $20,9 \%$. As infecções de maior gravidade ocorrem em pacientes com IRIC 3 e a vigilância pós-alta é importante, na medida em que muitas infecções somente serão diagnosticadas após a alta hospitalar.
\end{abstract}

Unitermos: Ferida operatória; Infecção cirúrgica; Complicações pós-operatórias.

\section{INTRODUÇÃO}

A ISC (Infecção de Sítio Cirúrgico) representa 24\% das infecções hospitalares, segundo dados do projeto SENIC (Study on Efficacy of Nosocomial Infection Control), programa desenvolvido na América do Norte com o objetivo de avaliar a eficiência das Comissões de Controle de Infecções e Epidemiologia Hospitalar., ${ }^{1,2}$

O conhecimento da incidência e dos principais fatores de risco associados a essas complicações cirúrgicas, mediante utilização de um sistema de vigilância epidemiológica hospitalar eficiente, é importante na medida em que até 35\% delas podem ser prevenidas através da implementação de ações de controle de ISC. ${ }^{1}$

O objetivo do presente trabalho é verificar os índices de infecção em pacientes operados no Hospital Geral César Cals (HGCC), Fortaleza-CE, entre janeiro de 1996 e janeiro de 1998, estratificados conforme o IRIC previsto pelo Sistema
NNISS e observar o tipo de infecção ocorrido nesses diferentes grupos.

\section{PACIENTES E MÉTODOS}

Foram monitorizados para infecção de sítio cirúrgico 2.149 pacientes operados no Serviço de Cirurgia do HGCC, pelo período de janeiro de 1996 a janeiro de 1998.

A vigilância epidemiológica foi dirigida para o sítio cirúrgico, na opção detalhada, com o método de coleta de dados baseado na busca ativa. As definições estavam padronizadas conforme recomendação do CDC- $1992^{4}$ e a metodologia utilizada foi aquela proposta pelo Sistema NNISS, ${ }^{5} \mathrm{com}$ modificações.

Ingressavam no protocolo pacientes NNISS (data de admissão e alta na instituição não coincidentes e sem doença crônica como causa da sua internação) e que se submeteram a um procedimento cirúrgico NNISS (com única entrada ao

1.Preceptor do Programa na Área de Cirurgia Geral e Preceptor-Chefe de Residência Médica do Hospital Geral César Cals - HGCC Secretaria de Saúde do Estado do Ceará - SESA. Chefe do Serviço de Controle de Infecção Hospitalar - SCIH-HGCC.

Vice-Mestre do Capítulo do Ceará do CBC. Membro da Surgical Infection Society - SIS-USA.

2. Médico Residente R3 de Cirurgia Geral do HGCC-SESA-CE.

3. Enfermeira do Serviço de Controle de Infecção Hospitalar do HGCC-SESA-CE e Especialista em Enfermagem Médico-Cirúrgica pela

Universidade Estadual do Ceará - UECE.

Recebido em 1/6/98

Aceito para publicação em 11/1/99

Trabalho realizado no Hospital Geral Cesar Cals - HGCC - Secretaria de Saúde do Estado do Ceará - SESA. 
Centro Cirúrgico onde o cirurgião realizava o mínimo de uma incisão através da pele ou mucosa e essa era fechada ainda na sala de operação).

Na confecção das taxas de ISC consideraram-se no numerador, inclusive, os casos diagnosticados no ambulatório de egressos e no denominador os procedimentos cirúrgicos pertencentes às categorias de cirurgia geral (CGER), urológica (CURO), torácica (CTOR), plástica (CPLA) e vascular (CVAS) de acordo com o quadro 1.

Quadro 1

Cirurgias monitorizadas e respectivo cut point

\begin{tabular}{|c|c|c|c|}
\hline Categoria & Procedimento & Descrição & Cut Poin \\
\hline \multirow[t]{18}{*}{ CGER } & ESOF(ogit) & Esofagectomia parcial/total & \\
\hline & GAST(gast) & Gastrectomia parcial/total & 31 \\
\hline & APEN(appy) & Apendicectomia & \\
\hline & COLE(chol) & Colecistectomia & \\
\hline & VBFP(coli) & Anastomose biliodigestiva & 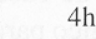 \\
\hline & & Papilotomia/papiloesfincteroplastia & \\
\hline & & Hepatectomia parcial & 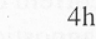 \\
\hline & & Pancreatectomia parcial & 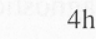 \\
\hline & ESPL(sple) & Esplenectomia & 21 \\
\hline & DELG(sb) & Enterectomias & \\
\hline & & Outras cirurgias do intestino delgado & \\
\hline & HERN(her) & Herniorrafia inguinal & \\
\hline & & Herniorrafia epigástrica/umbilical & \\
\hline & $\mathrm{COLO}$ (colo) & Colectomia parcial/total & \\
\hline & LAPE(xlap) & Laparotomia exploradora & \\
\hline & OUTR & Histerectomia abdominal & \\
\hline & & Mastectomia & \\
\hline & ENDC(oes) & Tireoidectomia $\mathrm{parcial} /$ total & \\
\hline CURO & MASC(prst) & Prostatectomia suprapúbica & \\
\hline \multirow{3}{*}{$\begin{array}{r}\text { CTOR } \\
\text { (thor) }\end{array}$} & & & \\
\hline & CTOR & Lobectomia & \\
\hline & & Decorticação pulmonar & \\
\hline \multirow[t]{2}{*}{$\begin{array}{l}\text { CPLA } \\
(\text { oskn) }\end{array}$} & CPLA & Mamoplastia & \\
\hline & & Dermolipectomia & \\
\hline $\begin{array}{l}\text { CVAS } \\
\text { (ocvs) }\end{array}$ & CVAS(vs) & Safenectomia & \\
\hline
\end{tabular}

Fonte: Am J Med, Sep 16, 1991; 91(suppl 3B): 152S-157S

(Modificado)

Os elementos necessários à elaboração do IRIC eram registrados e condensados na própria folha de descrição operatória, evitando assim o sub-registro (Quadro 2).

A análise estatística da relação entre o IRIC e o tipo de infecção foi pelo teste de independência, Qui-quadrado de Pearson, utilizando nível de significância de 5\%. A comparação entre as proporções de infecção nos diversos IRIC's em

Quadro 2

Dados da folha de descrição operatória

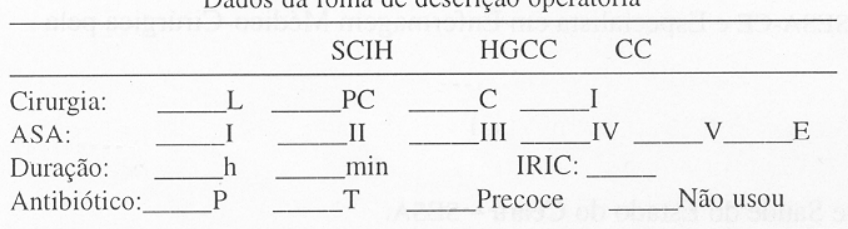

relação a outras publicações foi feito pelo teste binomial com nível de significância de 5\%, bicaudal.

\section{RESULTADOS}

Dos 2.149 pacientes NNISS operados, 120 evoluíram com infecção, correspondendo a uma taxa geral de infecção de sítio cirúrgico de 5,6\%. Estratificando-se pelo Índice de Risco de Infecção Cirúrgica - IRIC, essas taxas correspondem a $3,2 \%, 7,4 \%, 16,6 \%$ e $20,9 \%$, respectivamente para os IRICs $0,1,2$ e 3 (Tabela 1 ).

Do total de procedimentos cirúrgicos NNISS acompanhados no Serviço, 1.478 ou $68,8 \%$ pertenceram ao IRIC 0 . Nesse grupo, 47 pacientes infectaram e o tipo de infecção predominante foi a incisional superficial, com 45 casos.ou $95,7 \%$. Já no IRIC 3, tivemos 67 cirurgias ou 3,1\% do total, com 14 infectados, sendo que, desses, 10 ou 71,4\% evoluíram com infecção do tipo incisional profunda ou órgão/cavidade (Tabela 1).

Tabela 1

Incidência de infecção de sítio cirúrgico por IRIC - jan/96 a jan/98

\begin{tabular}{c|r|r|c|c|c|c|c|r}
\hline Indicador & \multirow{2}{*}{$\mathrm{N}^{\circ}$ de } & \% do & \multicolumn{6}{|c}{ Tipo de Infecção } \\
\cline { 5 - 10 } IRIC & Cirurgias & Total & Superficial & $\%$ & Prof + Org/Cav & $\%$ & Total & Tx. ISC \\
\hline 0 & 1.478 & 68,8 & 45 & 95,7 & 2 & 4,3 & 47 & 3,2 \\
1 & 447 & 20,8 & 24 & 72,7 & 9 & 27,3 & 33 & 7,4 \\
2 & 157 & 7,3 & 18 & 69,2 & 8 & 30,8 & 26 & 16,6 \\
3 & 67 & 3,1 & 4 & 28,6 & 10 & 71,4 & 14 & 20,9 \\
\hline Total & 2149 & 100 & 91 & 75,8 & 29 & 24,2 & 120 & 5,6 \\
\hline
\end{tabular}

Fonte: SCIH do HGCC

No ano de 1997 foram realizadas 240 colecistectomias com oito infectadas, o que representa uma taxa global de ISC de $3,3 \%$. Estratificando-se por via de acesso, essa taxa foi de $4,9 \%$ para as convencionais e $1 \%$ para as laparoscópicas. Estratificando-se as 143 colecistectomias convencionais por IRIC, a taxa de ISC do grupo 0 foi de $4,3 \%$ e do grupo 2 de $16,7 \%$. Nenhuma colecistectomia classificou-se no IRIC 3 (Tabela 2).

Tabela 2

Incidência de infecção de sítio cirúrgico em colecistectomias no ano de 1997

\begin{tabular}{c|c|c|c|r|r|r|r|r|r}
\hline & \multicolumn{3}{|c|}{ Convencional } & \multicolumn{4}{c|}{ Laparoscópica } & \multicolumn{3}{c}{ Todas } \\
\hline IRIC & Cir & Inf & $\%$ & Cir & Inf & $\%$ & Cir & Inf & $\%$ \\
\hline 0 & 92 & 4 & 4,3 & 97 & 1 & 1,0 & 189 & 5 & 2,6 \\
1 & 45 & 2 & 4,4 & 0 & 0 & 0 & 45 & 2 & 4,4 \\
2 & 6 & 1 & 16,7 & 0 & 0 & 0 & 6 & 1 & 16,7 \\
3 & 0 & 0 & 0 & 0 & 0 & 0 & 0 & 0 & 0 \\
\hline Total & 143 & 7 & 4,9 & 97 & 1 & 1,0 & 240 & 8 & 3,3 \\
\hline
\end{tabular}

Fonte: SCIH do HGCC

Quanto às hérnias incisionais, inguinais, epigástricas, umbilicais e outras, de 147 operadas no mesmo período, en- 
Gráfico 1. Vigilância Epidemiológica Pós-alta Jan-96 a Jan-98

$67,5 \%$

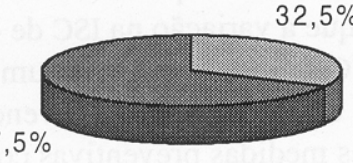

$\square$ Ambulatório
$\square$ Hospital

$N=2149(n=120)$

Fonte: SCIH do HHGCC

contramos taxa global de ISC em torno de 4,8\%. De 18 hérnias incisionais do IRIC 0 , constatamos uma infecção, o que equivale a uma taxa de ISC de 5,6\% para esse grupo específico. Das 120 infecções ocorridas em 2.149 cirurgias realizadas no período de janeiro de 1996 a janeiro de 1998, 39 (32,5\%) foram diagnosticadas no àmbulatório de egressos (Gráfico 1).

\section{DISCUSSÃO}

A infecção de ferida operatória é a complicação cirúrgica mais comum, manifesta-se comumente pela presença de pus na incisão e se apresenta com uma morbi-mortalidade muito variável. ${ }^{6}$ A denominação "ferida operatória infectada" refere-se geralmente àquelas localizadas no tecido subcutâneo imediatamente abaixo da linha de sutura, tecidos moles profundos, inclusive abaixo da aponeurose, porém não contempla aquelas complicações infecciosas graves desenvolvidas no órgão ou cavidade manipulados durante o ato operatório.

Em 1992, o Centro para Controle de Doenças (CDC) de Atlanta-USA, acatando sugestão de comitê formado por membros de importantes entidades científicas americanas (The Surgical Wound Infection Task Force) substituiu a denominação Infecção de Ferida Operatória por Infecção de Sítio Cirúrgico (ISC) ${ }^{4,7}$ e criou uma classificação que identificava o sítio anatômico acometido.

Assim, as diversas complicações infecciosas associadas aos atos operatórios que envolviam pele e subcutâneo passaram a ser definidas como Incisionais Superficiais, aquelas de fáscias e músculos como Incisionais Profundas e as que envolviam órgãos ou cavidades manipuladas durante o procedimento cirúrgico como ISC Órgão/Cavidade.

Com relação ao risco de desenvolvimento dessas infecções, a determinação de um índice que viesse a predizer esse risco começou a ser perseguido em 1964 pelo Conselho Nacional de Pesquisa Americano, ${ }^{8,9,10,11}$ com a tradicional classificação das cirurgias nas categorias Limpa, Potencialmente Contaminada, Contaminada e Infectada. Em 1970, o Projeto SENIC (Study on the Efficacy of Nosocomial Infection Control), ${ }^{12,13}$ utilizando dez fatores de risco e a técnica estatística do sistema CHAID (chi-square automatic interation detection), desenvolveu um índice cuja operacionalização ficava comprometida pela dificuldade em dimensionar todos aqueles fatores de risco ${ }^{14}$ Em 1985, Haley et al ${ }^{15}$ simplificaram essa metodologia, reduzindo para quatro fatores - grau de contaminação, operação abdominal, duração superior a duas horas e paciente com mais de três diagnósticos por ocasião da alta. Recentemente, Culver ${ }^{16}$ modificou esse índice, substituindo o último critério por um escore de avaliação préoperatória com base na classificação da Sociedade Americana de Anestesiologistas (ASA). Baseados nessa nova metodologia, os pesquisadores do Sistema NNISS (National Nosocomial Infections Surveillance System) - CDC desenvolveram um Índice de Risco de Infecção Cirúrgica (IRIC) que melhor prediz o risco de infecção e considera os fatores mais importantes como cirurgia contaminada ou infectada, pacientes ASA.III, IV ou V e a duração da cirurgia maior que o cut point, esse baseado no tempo médio das cirurgias analisadas pelo NNISS. De acordo com a presença ou ausência desses fatores, é atribuído o escore 1 ou 0, respectivamente (Quadro 3).

Quadro 3

Determinação do índice de risco

\begin{tabular}{lcc}
\hline Fatores de Risco & Paciente A & Paciente B \\
\hline Tipo de Cirurgia & Limpa (escore 0) & Contaminada (escore 1) \\
Duração (Cut Point) & Menor (escore 0) & Maior (escore 1) \\
ASA & II (escore 0) & IV (escore 1) \\
\hline IRIC & 0 & 3 \\
\hline
\end{tabular}

No Brasil, dados extraídos de observações clínicas assinalam uma ocorrência de infecção de ferida operatória em torno de $11 \% .^{10,17}$ De 1991 a 1995, no Serviço de Cirurgia do HGCC, época em que as definições do CDC-1992 não eram adotadas e o risco de infecção baseava-se no tradicional grau de contaminação das cirurgias, essa incidência foi de 8,3 infectados por cem operados. Na interpretação desses dados, mesmo quando estratificados por tipo de cirurgia, as informações sobre natureza e morbi-letalidade dessas complicações eram limitadas.

No presente trabalho, usando uma metodologia diferente, a NNISS, tivemos uma taxa global de ISC de 5,6\%, que, estratificada pelos IRICs $0,1,2$ e 3, facilita estudos comparativos entre instituições, melhor prediz o risco de infecção nos pacientes operados e confirma a relação entre IRIC e os tipos de ISC, a um nível de significância de 5\% (Tabela 1). A variação de 3,2\% nos pacientes do IRIC 0 para $20,9 \%$ nos pacientes do IRIC 3, onde os três fatores de risco estavam presentes, comprova a influência desses fatores na incidência de infecção e a sua conseqüente importância na elaboração do referido índice.

Starling et al, ${ }^{5}$ monitorizando 11.837 pacientes operados em cinco hospitais brasileiros no período compreendido entre julho de 1991 e setembro de 1993 , e Culver et al, ${ }^{16}$ monitorizando 84.691 pacientes operados em 44 hospitais americanos, de janeiro de 1987 a dezembro de 1990, ambos utilizando-se 
Tabela 3

Incidência de infecção de sítio cirúrgico por IRIC e autores

\begin{tabular}{|c|c|c|c|c|c|c|c|}
\hline \multirow[b]{2}{*}{ Autores } & \multirow{2}{*}{$\begin{array}{l}\text { Cirurgias } \\
\text { Realizadas }\end{array}$} & \multirow{2}{*}{$\begin{array}{c}\text { Cirurgias } \\
\text { Infectadas }\end{array}$} & \multicolumn{5}{|c|}{ Taxas de ISC } \\
\hline & & & IRIC 0 & IRIC 1 & IRIC 2 & IRIC 3 & Global \\
\hline $\begin{array}{l}\text { Culver-USA } \\
01 / 87 \text { a } 12 / 90\end{array}$ & $\begin{array}{c}84.691 \\
\text { (Multicêntrico) }\end{array}$ & $2 . .376$ & $1,50 \%$ & $2,90 \%$ & $6,80 \%$ & $13 \%$ & $2,80 \%$ \\
\hline $\begin{array}{l}\text { Starling - } \\
\quad \text { Brasil } \\
\text { 07/91 a 09/93 }\end{array}$ & $\begin{array}{c}11 . .837 \\
\text { (Multicêntrico) }\end{array}$ & 438 & $1,50 \%$ & $4,40 \%$ & $11,10 \%$ & $19,80 \%$ & $3,70 \%$ \\
\hline $\begin{array}{l}\text { Lemos - Brasil } \\
01 / 96 \text { a 01/98 }\end{array}$ & $\begin{array}{r}2.149 \\
(\mathrm{HGCC})\end{array}$ & 120 & $3,20 \%$ & $7,40 \%$ & $16,60 \%$ & $20,90 \%$ & $5,60 \%$ \\
\hline
\end{tabular}

Lemos, $F N-1998$

da metodologia NNISS, relataram taxas de ISC menores, em especial para os grupos de baixo risco (Tabela 3 ).

Em uma análise comparativa, a um nível de significância de $5 \%$, os valores observados nos IRIC's 0,1 e 2 de Lemos (Brasil) são estatisticamente diferentes dos encontrados em Culver (USA) e Starling (Brasil). No IRIC 3, diferente dos outros, os índices de infecção não são considerados estatisticamente diferentes.

Permanência pré-operatória prolongada, cuja associação com a ocorrência de ISC está bem definida na literatura, ${ }^{11} \mathrm{e}$ má nutrição, que parece interferir também na incidência de ISC ${ }^{18}{ }^{18}$ são características marcantes da clientela do HGCC. Esses fatores, associados a outra característica tradicional do cirurgião do HGCC em proceder a abertura parcial da incisão cirúrgica sob pretexto de prevenir possíveis seromas, conduta essa hoje coibida pelo SCIH mas que levou esse Serviço a diagnosticar epidemiologicamente esses casos como infectados, podem ter contribuído para uma maior taxa de ISC em Lemos (Brasil).

Dos 120 pacientes infectados, 75,8\% tiveram ISC incisional superficial, de menor gravidade e tratamento geralmente restrito ao local da incisão. Esse tipo de infecção é mais comum nos pacientes pertencentes ao IRIC 0. Já nos do IRIC 3, 71,4\% contraíram ISC do tipo incisional profunda ou órgão/cavidade, essas comportando uma morbi-letalidade maior e, conseqüentemente, exigindo terapêutica mais complexa (Tabela 1).

Importante ressaltar que a variação na ISC de 4,3\% no IRIC 0 para $16,7 \%$ no IRIC 2 observadas para um mesmo procedimento cirúrgico - colecistectomia convencional certamente influenciará nas medidas preventivas como, por exemplo, recomendação de uso da antibioticoprofilaxia cirúrgica para os pacientes pertencentes ao IRIC 2 e não para os pacientes do IRIC $0 .{ }^{19}$

Um tipo específico e grave de infecção chamada Fascite Necrotizante (FN) Pós-Operatória, diagnosticada no HGCC conforme critérios preconizados por Fisher et al, ${ }^{20}$ não foi incluído no presente estudo. Tivemos três casos no período e, por se constituir num grupo muito específico em termos de etiopatogenia, fisiopatologia, diagnóstico e tratamento, o SCIH tem como rotina classificá-los separadamente.

Os dados relatados estariam subestimados não fosse a vigilância pós-alta desses pacientes, na medida em que $32,5 \%$ das infecções foram detectadas no ambulatório de egressos.

Um serviço de cirurgia que colete os seus dados baseado nas novas definições para infecção de sítio cirúrgico recomendadas pelo CDC, que elabore um Índice de Risco de Infecção Cirúrgica-IRIC conforme recomendado pelos pesquisadores do Sistema NNISS e que disponha de um ambulatório de egressos, onde os pacientes de alta hospitalar são monitorizados para infecção de sítio cirúrgico, certamente terá indicadores epidemiológicos de infecção cirúrgica mais fidedignos, para orientar os cirurgiões nas suas ações de controle e prevenção das infecções, bem como para permitir a comparação dessas taxas entre eles e entre instituições diferentes.

\begin{abstract}
Patients undergoing surgery are at risk of surgical site infection-SSI. According to data from the National Nosocomial Infection Surveillance System-NNISS, in the United States surgical infections are the second most common type of nosocomial infection. The aim of this study is to assess surgical-site infection rates for patients operated in a general hospital in Northeasteen Brazil, break them down according to the Surgical Infection Risk Index-SIRI, and then classify such infections according to their gravity. A prospective evaluation was conducted of 2,149 surgical patients between January 1996 and January 1998, at the Cesar Cals General Hospital, using the methodology of the NNISS, adapted to our hospital. Definition standards were those recommended by Center for Disease Control-CDC-1992. Statistical analysis was based on Qui-square test of Pearson. Of the 2,149 NNISS patients operated, 120 developed infection, thus corresponding to a general surgicalsite infection rate of 5.6\%. Broken down according to the Surgical Infection Risk Index-SIRI, such rates correspond to 3.2\%, $7.4 \%, 16.6 \%$ and $20.9 \%$ for the risk-index groups $0,1,2$ and 3, respectively. Of all NNISS surgical procedures monitored at the Service, 1,478 (68.8\%) were included in SIRI 0. In that group, 47 patients developed infection, the superficial incisional being the predominant infection type, which accounted for 45 cases (95.7\%). In contrast, in SIRI 3, out of the 67 surgeries (3.14\% of the total), 14 developed infection, 10 of which (71.4\%) were deep incisional or organ/cavity infection. Out of the 120 infections occurred in 2, 149 surgeries performed in the period January 1996-January 1998, 39 (32.5\%) were diagnosed in outpatient wards. The rates obtained under NNISS recommendations are trustworth and allow comparative studies among different institutions.
\end{abstract}

Key Words: Operative wound; Surgical infection; Postoperative complications. 


\section{REFERÊNCIAS}

1. Haley RW, Culver DH, White JW, et al - The efficacy of infection surveillance and control programs in preventing nosocomial infections in US hospital. Am J Epidemiol 1985;121:182.

2. Haley RW, Gaynes RP, Aber RC, et al - "Surveillance of nosocomial infections". In Bennett JV et al.: Hospital Infections. $3^{\text {rd }}$ Ed. Boston, MA: Little, Brown and Co, 1992:101.

3. Martone WJ, Jarvis WR, Culver DH, et al - "Incidence and nature of endemic and epidemic nosocomial infections". In Bennett JV, et al.: Hospital Infections. $3^{\text {rd }}$ Ed. Boston, MA: Little, Brown and Co, 1992:597.

4. Horan TC, Gaynes RP, Martone WJ, et al - CDC definitions of nosocomial surgical site infections, 1992: A modificacion of CDC definitions of surgical wound infections. Infect Control Hosp Epidemiol, 1992 Oct; 13(10): 606-608.

5. Starling CEF, Almeida FF, Pinheiro SMC et al - "Vigilância epidemiológica de infecções hospitalares: Metodologia NNIS-CDC aplicada em hospitais brasileiros". In Ferraz EM: Infecção em cirurgia.

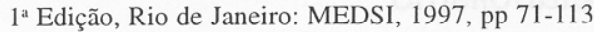

6. Bacelar TS, Ferraz EM - "Infecção hospitalar em cirurgia”. In Zilberstein B, Cecconello I, Félix VN, et al - Infecção em cirurgia do aparelho digestivo. $1^{\text {a }}$ Edição. São Paulo: Robe Editora, 1994, pp 462-489.

7. Garner JS, Jarvis WR, Emori TG, et al - CDC definitions for nosocomial infections, 1988.American Journal of Infection Control, 1988 Jun; 6(3):128-140.

8. Sawyer RG, Pruett TL - Wound Infection. Surg Clin North Am 1994 Jun; 4(3):519-536.

9. Condon RE, Wittmann DH - "Surgical Infection." In: Morris PJ and Malt RA - Oxford text book of surgery. Oxford Medical Publications, 1994 , v. 1, cap. 2, pp 27-50.

10. Gribaum RS - "Infecção do sítio cirúrgico e antibioticoprofilaxia em cirurgia." In: Rodrigues EAC, Mendonça JS, Amarante JMB, et al Infecções hospitalares-prevenção e controle. São Paulo: Sarvier, 1997, pp 149-167.

11. Cruse PSG, Foord M - The epidemiology of wound infection. Surg Clin North Am 1980; 60: 27- 40.

12. Mayhall CG - "Surgical infections including Burns". In Wenzel RP: Prevention and Control of Nosocomial Infections. $2^{\text {nd }}$ Edition. Baltimore: Williams \& Wilkins, 1993, pp 614-654.

13. Ferraz AAB, Ferraz EM, Bacelar TS - "Infecção da ferida cirúrgica". In Ferraz EM: Infecção em cirurgia. 1 ${ }^{\text {a }}$ Edição. Rio de Janeiro: MEDSI, 1997, pp 267-275.
14. Robson MC - Wound Infection:A failure of wound healing caused by na imbalance of bacteria. Surg Clin North Am 1997 Jun;77(3): 637-649.

15. Hooton TM, Haley RW, Culver DH, et al - The joint association of multiple risk factors with the occorrence of nosocomial infection. Am J Med 1981;70:960.

16. Culver DH, Horan TC, Gaynes RP, et al - Surgical wound infection rates by wound class, operative procedure, and risk index. Am J Med Sep 16, 1991;91(suppl 3B):152S-157S.

17. Ferraz EM, Bacelar TS, Aguiar JLA, et al - Controle de infecção em cirurgia geral: resultados de um estudo prospectivo de 13 anos e de 17503 operações. Rev Col Bras Cir 1992;19: 169-74.

18. Garner JS - CDC guideline for prevention of surgical wound infections, 1985. Infect 'Cont Hosp Epidemiol 1986;7:193

19. Rocha PRS, Almeida, SR, Sanches, MD - "Infecção de sítio cirúrgico: fatores de risco e profilaxia”. In: Rocha PRS, Rodrigues MAG: Complicações em cirurgia do aparelho digestivo. São Paulo: Atheneu, Ano IV-Volume II-1998, pp 51-73.

20. Fisher JR, Conway MI, Takeshita RT - Necrotizing fasciitis. Importance of roentgenographic studies for soft-tissue gas. JAMA 1979;241:803.

\section{ENDERECCO PARA CORRESPONDÊNCIA}

Dr. Francisco Ney Lemos

Rua República do Líbano, 300/102-A

60160-140, Meireles, Fortaleza-CE

E-mail: fneyl@sec.secrel.com.br 